\title{
Strongly-Connected Outerplanar Graphs with Proper Touching Triangle Representations
}

\author{
J. Joseph Fowler \\ Department of Computer Science, University of Arizona, Tucson, AZ, USA \\ fowler@email.arizona.edu
}

\begin{abstract}
A proper touching triangle representation $\mathcal{R}$ of an $n$-vertex planar graph consists of a triangle divided into $n$ non-overlapping triangles. A pair of triangles are considered to be adjacent if they share a partial side of positive length. Each triangle in $\mathcal{R}$ represents a vertex, while each pair of adjacent triangles represents an edge in the planar graph. We consider the problem of determining when a proper touching triangle representation exists for a strongly-connected outerplanar graph, which is biconnected and after the removal of all degree- 2 vertices and outeredges, the resulting connected subgraph only has chord edges (w.r.t. the original graph). We show that such a graph has a proper representation if and only if the graph has at most two internal faces (i.e., faces with no outeredges).
\end{abstract}

\section{Introduction}

Although the node-link model has been the traditional form of drawing a planar graph $G(V, E)$, many application areas demand alternate models of representing graphs, such as polygon edge-contact representations. Here vertices are represented by simple polygons and edges are represented by adjacent polygons that have at least a partial side in common. As pointed out by de Fraysseix et al. [2], one can easily find such a representation of any planar graph with non-convex polygons with complexity as high as $|V|-1$, where much area is unused leading to many gaps and holes within the representation. Recently, convex hexagons have been shown to always be sufficient in producing hole-free representations [1], although, 6-sided polygons are sometimes necessary. The problem thus arises in determining which classes of planar graphs can be represented by polygons with fewer than six sides.

In this context, we focus on the case of minimal polygonal complexity, where all the representing polygons are triangles. Specifically, an $n$-vertex touching triangle graph (TTG) has a representation $\mathcal{R}$ where each vertex is represented by one of $n$ nonoverlapping triangles and each edge is represented by a pair of adjacent triangles in $\mathcal{R}$. Again, triangles are only considered to be adjacent if they share at least a partial side of positive length. Thus, pairs of triangles having only point contacts are not considered to be adjacent, and hence, do not represent edges.

The most natural looking edge-contact representations have triangular boundaries where their interiors contains no gaps or holes. If this is the case, then $\mathcal{R}$ is a proper TTG representation, and the graph is a proper TTG. Visually, $\mathcal{R}$ can be thought of as a triangle that has been subdivided into $n$ non-overlapping triangles, where adjacent

S. Wismath and A. Wolff (Eds.): GD 2013, LNCS 8242, pp. 155-160, 2013.

(C) Springer International Publishing Switzerland 2013 


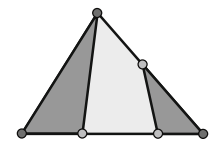

(a) Corner triangles

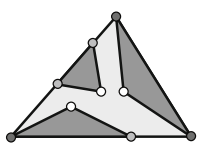

(b) Side triangles

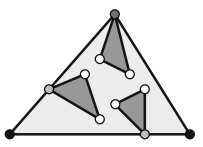

(c) Internal triangles

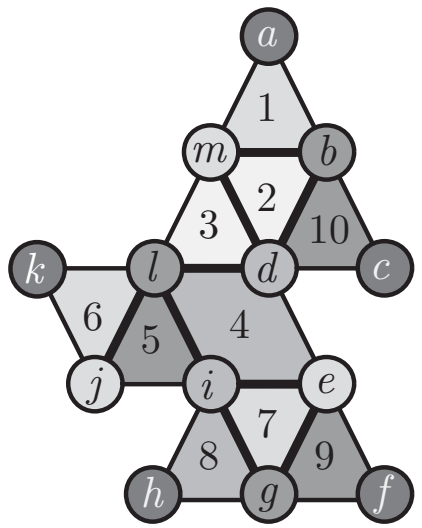

(d) Graph $G$

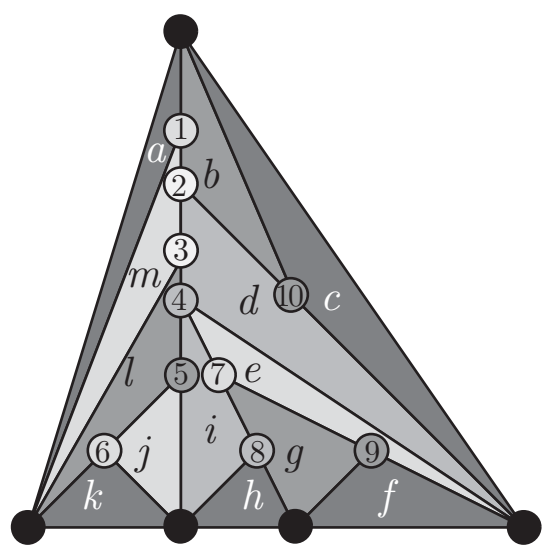

(e) Representation $\mathcal{R}$

Fig. 1. (a-c) Three types of representing triangles; (d) a strongly-connected outerplanar graph $G$ with two internal faces: 2 and 7; and (e) a proper TTG representation $\mathcal{R}$ of $G$

triangles do not necessarily share entire sides as they do in a triangulation. Not all planar graphs are TTGs [3], let alone proper. While it was also shown in [3] that all biconnected outerplanar graphs have hole-free TTG representations, their boundaries are not necessarily triangular, and hence, are not necessarily proper. This raises the question as to which outerplanar graphs have proper TTG representations.

A proper outerplanar TTG has several restrictions. Degree-1 vertices can only be represented by corner triangles of $\mathcal{R}$ with two edges along the boundary $T$ of $\mathcal{R}$, while degree-2 vertices can also be represented by side triangles of $\mathcal{R}$ with one side along $T$. All other vertices are represented by internal triangles of $\mathcal{R} ; c f$. Figs. 1(a) $1(\mathrm{c})$,

A biconnected outerplanar graph $G$ is strongly-connected if after the removal of all degree-2 vertices and outeredges, the resulting connected subgraph only has chord edges (w.r.t. $G$ ) as in Fig. 1(d) Such graphs are not necessarily maximal. We characterize this graph class in terms of internal faces (i.e. faces with no outeredges) as follows:

(1) First, we construct proper TTG representations for strongly-connected outerplanar graphs, as in Fig. 1(e), using a chord-to-endpoint assignment that pairs each chord (except for one) with a distinct vertex that is also an endpoint of the chord.

(2) Second, we show that having at most two internal faces is sufficient when the graph is strongly-connected, since a chord-to-endpoint assignment exists in this case.

(3) Third, we finish our characterization by proving that having at most two internal faces is also necessary in order for $G$ to have a proper TTG representation.

To the best of our knowledge, the only other results specifically for proper TTGs are in [4], where a fixed-parameter tractable decision algorithm for 3-connected planar max-degree- $\Delta$ graphs is described, and where it it shown that planar 3-connected cubic graphs are proper TTGs. 


\section{Proper Strongly-Connected Outerplanar TTGs}

Let $G$ be a strongly-connected outerplanar graph. A strong reversed peeling order $\sigma$ is an ordering of the inner faces $F_{1}, \ldots, F_{q}$ of $G$ such that the subgraph $G_{i}=F_{1} \cup \cdots \cup F_{i}$ is also strongly-connected for each $i \in[1 \ldots q]$. We index the chords $\mathcal{C}=\left\{c_{2}, \ldots, c_{q}\right\}$ of $G$ by $\sigma$ such that $c_{i}$ is the chord of face $F_{i}$ that becomes an outeredge of $G_{i-1}$ when $F_{i}$ is "peeled" from $G_{i}$. Thus, $c_{i}$ is common to $F_{i^{\prime}}$ and $F_{i}$ for some $i^{\prime}<i$. Finally, a chordto-endpoint assignment $\tau: \mathcal{C}^{\prime} \rightarrow V^{\prime}$ of a strong reversed peeling order $\sigma$ assigns the chords $\mathcal{C}^{\prime}=\left\{c_{3}, \ldots, c_{q}\right\}=\mathcal{C} \backslash\left\{c_{2}\right\}$ to a subset of their endpoints $V^{\prime}=\left\{v_{3}, \ldots, v_{q}\right\}$ such that $\tau\left(c_{i}\right)=v_{i}$ is an endpoint of $c_{i}$ for each $i \in[3 . . q]$ where $v_{i} \neq v_{j}$ if $i \neq j$.

Claim 1. If $G$ is a strongly-connected outerplanar graph, then $G$ has a strong reversed peeling order $\sigma$.

Proof. Faces $F_{1}$ and $F_{2}$ of $\sigma$ can be any pair of adjacent faces. For $i \in[2 \ldots q-1]$, assume that subgraph $G_{i}=F_{1} \cup \cdots \cup F_{i}$, has the connected chord subgraph $H_{i}=c_{2} \cup \cdots \cup c_{i}$, where $c_{i}$ is the chord of face $F_{i}$ that was added to $G_{i-1}$. At least one outeredge of $G_{i}$ is a chord in $G$. Otherwise, the outerface of $G_{i}$, which is a separating cycle $C$ in $G$, would have a cut-vertex in $G$-all the cut edges in $G$ are chords, none of which can be in $C$-violating the biconnectivity of $G$. Since $G$ is strongly-connected, every chord $c$ in the outerface of $G_{i}$ must be incident to some chord of $H_{i}$. Thus, the next face $F_{i+1}$ in $\sigma$ can be any remaining face whose chord $c_{i+1}$ is an outeredge of $G_{i}$.

Lemma 2. If $G$ is a strongly-connected outerplanar graph for which there exists a chord-to-endpoint assignment $\tau$, then $G$ has a proper touching triangle representation.

Proof. Let $\sigma$ be the strong reversed peeling order of the faces $F_{1}, \ldots, F_{q}$ of $G$ given by the order of chords as assigned by $\tau$. We apply induction on $k$ and assume that we have a proper TTG representation $\mathcal{R}_{k}$ for $G_{k}$, and show how to modify $\mathcal{R}_{k}$ to obtain $\mathcal{R}_{k+1}$ for $G_{k+1}=G_{k} \cup F_{k+1}$. When $G_{1}$ has a single face $F_{1}$ with the edge $(u, v)$ connected by the chain of vertices $x_{1}, \ldots, x_{i}$, Fig. 2(a) gives a proper TTG representation $\mathcal{R}_{1}$ for $G_{1}$. Next, when $G_{2}$ has two faces with the common chord $(u, v)$ (where $y_{1}, \ldots, y_{j}$ forms a chain in $F_{2}$ ), Fig. 2(b) gives a proper TTG representation $\mathcal{R}_{2}$ for $G_{2}$ where the triangle $\triangle u$ representing $u$ in $\mathcal{R}_{1}$ was subdivided into a total of $j+1$ triangles.

For $k \in[2 . . q-1]$, we also assume by induction on $k$ that triangle $\triangle v_{k}$ representing $\tau\left(c_{k}\right)=v_{k}$ is a side triangle in $\mathcal{R}_{k}$. This holds in the base case of $k=2$, since $\mathcal{R}_{2}$ in

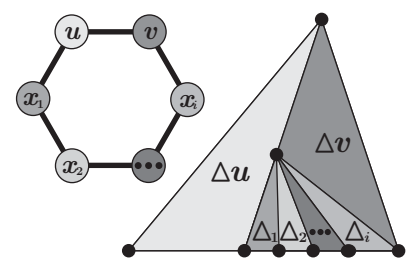

(a) $G_{1}$ and $\mathcal{R}_{1}$

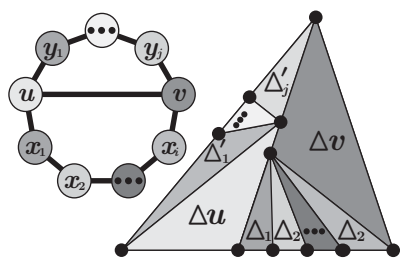

(b) $G_{2}$ and $\mathcal{R}_{2}$

Fig. 2. Proper TTG representations of strongly-connected outerplanar graphs with 1 or 2 faces 


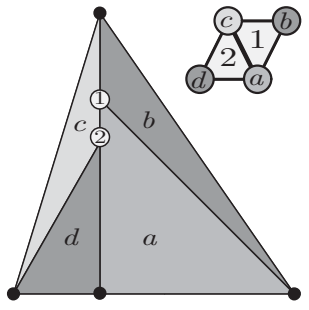

(a) $\mathcal{R}_{2}$ of $G_{2}$

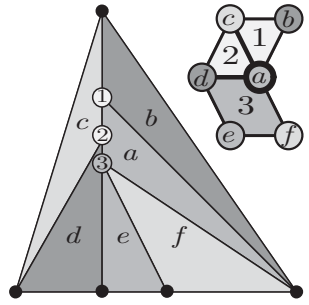

(b) $\mathcal{R}_{3}$ of $G_{3}$

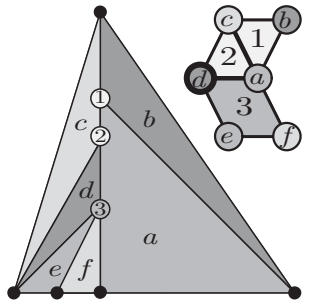

(c) $\mathcal{R}_{3}^{\prime}$ of $G_{3}$

Fig. 3. For chord $c_{3}=(a, d)$, either triangle $\triangle a$ or $\triangle d$ in $\mathcal{R}_{2}$ in (a) is divided into triangles $\triangle \tau\left(c_{3}\right), \triangle e, \triangle f$ to form $\mathcal{R}_{3}$ if $\tau\left(c_{3}\right)=a$ as in (b) or $\mathcal{R}_{3}^{\prime}$ if $\tau\left(c_{3}\right)=d$ as in (c)

Fig. 2(b) only has side triangles. This allows us to subdivide $\triangle v_{k}$ in $\mathcal{R}_{k}$ a total of $j_{k}$ times to obtain $\mathcal{R}_{k+1}$ in which an internal triangle now represents $v_{k}$ and $j_{k}$ new side triangles represent the $j_{k}$ degree- 2 vertices in $F_{k}$ that were added to $G_{k}$ to form $G_{k+1}$. Figure 3 illustrates this process. The chord $c_{3}=(a, d)$ in $G_{3}$ either has $\tau\left(c_{3}\right)=a$ or $\tau\left(c_{3}\right)=d$. This results in $\mathcal{R}_{3}$ in Fig. 3(b) (or in $\mathcal{R}_{3}^{\prime}$ in Fig. 3(c) after dividing the side triangle $\triangle a$ (or $\triangle d$ ) in $\mathcal{R}_{2}$ of $G_{2}$ in Fig. 3(a) into the internal triangle $\triangle a$ (or $\triangle d$ ) and the two side triangles $\triangle e$ and $\triangle f$. As a consequence, each chord endpoint $v_{k}$ has its representing triangle $\triangle v_{k}$ subdivided once, at which point $\triangle v_{k}$ is an internal triangle in $\mathcal{R}_{k^{\prime}}$ for $k^{\prime}>k$. However, this is not a problem in maintaining our inductive hypothesis for $k+1$ since $\tau$ assigns each chord to a distinct vertex in $G$.

Lemma 3. If $G$ is a strongly-connected outerplanar graph with at most two internal faces, then $G$ is a proper touching triangle graph.

Proof. We construct a chord-to-endpoint assignment $\tau$, where Lemma 2 then implies that $G$ is a proper TTG. If $G$ has no internal faces, its connected chord subgraph $H$ is acyclic, and hence, a tree. Let $\sigma$ be a strong reversed peeling order of faces $F_{1}, \ldots, F_{q}$ for $G$ given by Claim 1 Each face $F_{i}$ was picked so that its chord $c_{i}$ is a leaf edge in the subtree of chords $H_{i}=c_{2} \cup \cdots \cup c_{i}$ of $G_{i}$. Thus, we can assign $\tau\left(c_{i}\right)=u_{i}$, where $u_{i}$ is the endpoint of $c_{i}$ that is a leaf node in $H_{i}$, for $i \in[3 \ldots q]$. Both endpoints of the first chord $c_{2}$ are left unassigned by $\tau$.

If $G$ has one internal face $F$, we apply Claim 1 and assign chords as before with the following exceptions: We set $F_{1}=F$ and faces $F_{2}, \ldots, F_{j}$ as the adjacent faces of $F_{1}$, where face $F_{i+1}$ is incident to face $F_{i}$ for $i \in\{2 . . j-1\}$. Since chord $c_{j}$ forms the cycle $C$ (edges of $F_{1}$ ) when added to $H_{j}$, we can set $\tau\left(c_{j}\right)$ to be the common endpoint of the chords $c_{j}$ and $c_{2}$, which leaves one endpoint of $c_{2}$ unassigned.

Lastly, when $G$ has two internal faces, we apply Claim 1 as follows: We set $F_{1}$ and $F_{k}$ in $\sigma$ to be the two internal faces in $G$ such that $k$ is minimal. The chords of $G_{k}$ contain a path $p$ connecting $F_{1}$ to $F_{k}$. For $\tau$, we assign each chord of $p$ to the endpoint first encountered along $p$ so that $c_{2}$ and $c_{k}$ each have exactly one assigned endpoint. To $\sigma$ we add each remaining face $F_{i}$ adjacent first to $F_{1}$ (and then to $F_{k}$ ) starting from the endpoints of $p$ in cyclic order along each respective face. For $\tau$, we assign chord $c_{i}$ of each cycle to its newly added endpoint in $H_{i}$ - except for the last chord, call it $c^{\prime}$, in $F_{1}$ 


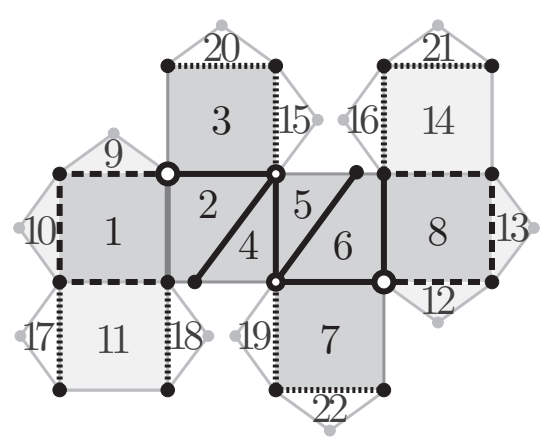

(a) Strong reversed peeling order $\sigma$

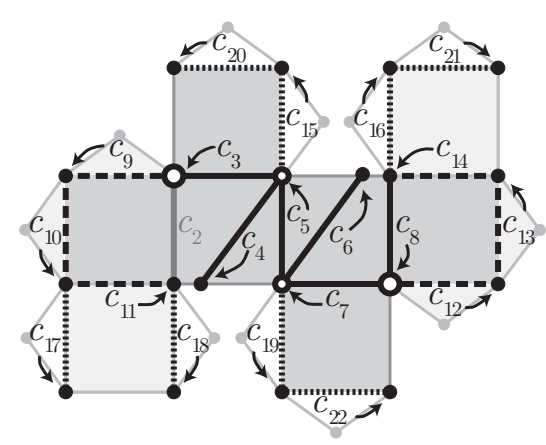

(b) Chord-to-endpoint assignment $\tau$

Fig. 4. Example of determining $\sigma$ and $\tau$ for strongly-connected outerplanar graph $G$ with two internal faces $F_{1}$ and $F_{8}$. Subgraph $G_{8}$ (dark gray faces) is the minimal strongly-connected subgraph containing both $F_{1}$ and $F_{8}$, whose chord subgraph $H_{8}$ (solid chords) is a caterpillar. Subgraph $G_{14}$ (light/dark gray faces) has faces $F_{9}, \ldots, F_{11}$ and $F_{12}, \ldots, F_{14}$ added in cyclic order along $F_{1}$ and $F_{8}$, resp., each starting from an endpoint of path $p$ (white vertices). The chords of path $p$ are assigned the endpoints first encountered along $p$ from $F_{1}$ to $F_{8}$. The dashed chords of $F_{1}$ and $F_{8}$ are assigned endpoints next along each cycle (starting from endpoints of $p$ ). Chord $c_{i}$ for $i \in\{4,6,15, \ldots, 22\}$ is assigned its endpoint that was not in the chord subgraph $H_{i-1}$.

(or in $F_{k}$ ). Given the greedy assignment of chords along $p, c^{\prime}$ has an available endpoint in common with $c_{2}$ in $F_{1}$ (or $c_{k}$ in $F_{k}$ ). Each remaining face can be added to $\sigma$ and have its chord assigned by $\tau$ as before. Figure 4 illustrates this procedure.

For the next lemma, we consider the representing dual graph $\mathcal{G}_{\mathcal{R}}$ (the graph formed by the representation $\mathcal{R}$ ) of a proper TTG $G$. With respect to the triangular boundary $T$ of a proper TTG representation $\mathcal{R}$, each vertex or edge of $\mathcal{G}_{\mathcal{R}}$ (common to one or more representing triangles) is either external if along the boundary $T$ or internal if inside $T$. Likewise, we term the faces of $\mathcal{G}_{\mathcal{R}}$ as being either external faces if they correspond to corner or side triangles or internal faces, otherwise. We have the following relationships between $G$ and $\mathcal{G}_{\mathcal{R}}$ : (1) each vertex $v$ in $G$ corresponds to a bounded triangular face $F_{v}$ in $\mathcal{G}_{\mathcal{R}}$ and (2) each bounded face $f$ in $G$ corresponds to an internal vertex $v_{F}$ in $\mathcal{G}_{\mathcal{R}}$.

Clearly, the angle of a vertex $v_{F}$ in $\mathcal{G}_{\mathcal{R}}$ along the face $F_{v}$ in $\mathcal{G}_{\mathcal{R}}$ can be at most $180^{\circ}$. If the angle is less than $180^{\circ}$, then $v_{F}$ is a corner of the triangular face $F_{v}$. Each internal vertex $v_{F}$ in $\mathcal{G}_{\mathcal{R}}$ has at most one $180^{\circ}$ angle since $\operatorname{deg}\left(v_{F}\right)>2$ in $\mathcal{G}_{\mathcal{R}}$. For example, the angle of vertex $v_{1}$ in $\mathcal{R}$ in Fig. $1(\mathrm{e})$ is $180^{\circ}$ for face $F_{b}$, but not for faces $F_{a}$ and $F_{m}$. Face $F_{v}$ in $\mathcal{G}_{\mathcal{R}}$ representing $v$ has exactly three vertices (each with an angle less than $180^{\circ}$ ) if either (i) $F_{v}$ is an external face where $\operatorname{deg}(v)=2$ or (ii) $F_{v}$ is an internal face where $\operatorname{deg}(v)=3$. Otherwise, $F_{v}$ has at least $\operatorname{ch}(v)=\max \{\operatorname{deg}(v)-3,0\}$ vertices in $\mathcal{G}_{\mathcal{R}}$ whose internal angles are $180^{\circ}$ along the boundary of $F_{v}$. These correspond to $\operatorname{ch}(v)$ faces in $G$ that are incident to $v$.

We denote $\operatorname{ch}(v)$ as the charge of $v$ since each incident face $F$ in $G$ can dissipate at most one charge from $v$. This is done by having the internal angle for $v_{F}$ be $180^{\circ}$ along the face $F_{v}$ representing $v$ in $\mathcal{G}_{\mathcal{R}}$. However, face $F$ can dissipate at most one charge from an incident vertex, since $v_{F}$ has at most one $180^{\circ}$ angle. For example, vertex $d$ in 
Fig. 1(d) has charge $c h(d)=2$ dissipated by faces $F_{3}$ and $F_{10}$, where the corresponding internal vertices $v_{3}$ and $v_{10}$ have $180^{\circ}$ angles for face $F_{d}$ in Fig. $1(\mathrm{e})$.

Thus, in order for $G$ to be a TTG, there must exist a discharge function, $\pi: \mathcal{F}^{\prime} \rightarrow V$ that assigns a subset faces $\mathcal{F}^{\prime} \subseteq \mathcal{F}$ (the faces of $G$ ) to incident vertices to fully dissipate the total charge $\operatorname{ch}(G)=\sum_{v \in V(G)} \operatorname{ch}(v)$ of $G$. Hence, $G$ cannot be a proper TTG graph if $a v(G)=q-\operatorname{ch}(G)<0$, where $q=|\mathcal{F}|$ and $a v(G)$ is the availability of $G$.

Lemma 4. If $G$ is a strongly-connected outerplanar graph with more than two internal faces, then $G$ cannot be a proper touching triangle graph.

Proof. We apply Claim 1 to get a strong reversed peeling order $\sigma$ of the faces $F_{1}, \ldots, F_{q}$ of $G$, where the subgraph $G_{i}=F_{1} \cup \cdots \cup F_{i}$ is strongly-connected. If $G_{i}$ does not contain any internal faces, then $H_{i}$ is a tree. When peeling face $F_{i}$ from $G_{i}$ to obtain $G_{i-1}$, chord $c_{i}$ cannot have both of its endpoints $u_{i}$ and $v_{i}$ in $H_{i-1}$. Otherwise, $c_{i}$ would form a cycle in $H_{i}$. Thus, one endpoint $v_{i} \notin H_{i-1}$ of $c_{i}$ has $\operatorname{deg}\left(v_{i}\right)=2$ in $G_{i-1}$. While both degrees of $u_{i}$ and $v_{i}$ increased by 1 in going from $G_{i-1}$ to $G_{i}$, only $\operatorname{deg}\left(u_{i}\right)>3$ in $G_{i}$, while $\operatorname{deg}\left(v_{i}\right)=3$ in $G_{i}$. Thus, the total charge $\operatorname{ch}\left(G_{i}\right)=\operatorname{ch}\left(G_{i-1}\right)+1$ increases by one, so that the availability $a v\left(G_{i}\right)=a v\left(G_{i-1}\right)$ remains constant.

If $G_{i}$ contains a new internal face $C$ that $G_{i-1}$ does not, then both endpoints $u_{i}$ and $v_{i}$ of $c_{i}$ must be in $H_{i-1}$ in order for $c_{i}$ to form the new cycle $C$ in $H_{i}$. Hence, both $\operatorname{deg}\left(u_{i}\right)>3$ and $\operatorname{deg}\left(v_{i}\right)>3$ in $G_{i}$, so that $\operatorname{ch}\left(G_{i}\right)=\operatorname{ch}\left(G_{i-1}\right)+2$, and as a result, $a v\left(G_{i}\right)=a v\left(G_{i-1}\right)-1$ where the availability decreases by one.

Initially the availability is at most 2 , where $G_{2}$ has maximum degree 3 with the two faces $F_{1}$ and $F_{2}$ so that $a v\left(G_{2}\right)=2$. Consequently, $G$ can have at most two internal faces, before the availability drops below 0 , preventing it from being a TTG.

We conclude by combining Lemmas 3 and 4 to give the main theorem of the paper.

Theorem 5. A strongly-connected outerplanar graph $G$ has a proper touching triangle representation if and only if $G$ has at most two internal faces.

Acknowledgments. We thank our colleagues for helpful insights: Jawaherul Alam, Michael Kaufman, Stephen Kobourov, Martin Nöllenburg, Ignaz Rutter, Alexander Wolff, and many others.

\section{References}

1. Duncan, C.A., Gansner, E.R., Hu, Y.F., Kaufmann, M., Kobourov, S.G.: Optimal polygonal representation of planar graphs. Algorithmica 63(3), 672-691 (2012)

2. de Fraysseix, H., de Mendez, P.O., Rosenstiehl, P.: On triangle contact graphs. Combinatorics, Probability and Computing 3, 233-246 (1994)

3. Gansner, E.R., Hu, Y., Kobourov, S.G.: On touching triangle graphs. In: Brandes, U., Cornelsen, S. (eds.) GD 2010. LNCS, vol. 6502, pp. 250-261. Springer, Heidelberg (2011)

4. Kobourov, S.G., Mondal, D., Nishat, R.I.: Touching triangle representations for 3-connected planar graphs. In: Didimo, W., Patrignani, M. (eds.) GD 2012. LNCS, vol. 7704, pp. 199-210. Springer, Heidelberg (2013) 